\title{
Multidimensional Separations for Protein/ Peptide Analysis in the Post-Genomic Era
}

BioTechniques 32:898-911 (April 2002)

\author{
Hongbin Liu, Dayin Lin, and \\ John R. Yates III \\ The Scripps Research Institute, \\ La Jolla, CA, USA
}

\section{INTRODUCTION}

Advances in genome sequencing have allowed DNA sequence information to be compiled for a variety of organisms. The wealth of genomic information provides a parts list of the components of organisms but does not tell us how all the pieces or protein parts work together (12). The products of genes (i.e., proteins), rather than the genes themselves, carry out most biochemical processes in living organisms. Proteomics, or more precisely functional proteomics, has emerged as a new field to study protein function by examining the actions of proteins as part of a system or process. The systems or networks of proteins involved in biochemical processes or signal transduction pathways may be revealed through the examination of protein-protein interactions, changes in expression levels, or posttranslational modifications. Because of the inherent complexity of these systems, high-throughput technologies to separate proteins and peptides are needed. New developments in separation science, especially in the area of multidimensional protein/peptide separation, are evolving at a rapid pace and play a key role in proteomics research.

The proteome of a cell or microorganism, which on average consists of thousands to tens of thousands of proteins, invariably overwhelms the separation capacity of even the most sophisticated 1-D liquid chromatography (1DLC) system due to insufficient peak capacity. Based on Giddings' mathemat- ical models, the peak separation capacity of orthogonally coupled 2-D chromatographies approaches the product of the peak capacities of individual 1-D separations (14). A widely used 2-D separation technique in proteomics has been 2-D gel electrophoresis. Despite its popularity, this technology has several limitations. Proteins with extreme $\mathrm{pI}$ values (i.e., very basic and acidic proteins) and those with transmembrane domains cannot be separated by this method; at the same time, the process of in-gel digestion is laborious (11). To overcome these obstacles and advance proteomic research, several comprehensive, highthroughput 2-D liquid separation techniques have been developed in recent years $(26,39)$. Here we will review some online coupled 2-D liquid chromatography (2DLC) and online coupled liquid chromatography and capillary electrophoresis (LC-CE) techniques. When 2DLC techniques are combined with mass spectrometry, a virtual third separation and characterization dimension is added that greatly increases the power of the methods for proteomics. Multidimensional protein identification technology (MudPIT) is based on this concept and will also be reviewed.

\section{ONLINE COUPLED 2DLC}

In traditional 2DLC systems, liquid chromatographies with different retention properties are coupled in a "heartcutting" configuration to separate one or several components from a complex 
mixture $(1,3,31)$. In this type of configuration, only a portion of the effluent material from the first separation goes into the second; therefore, it is not a comprehensive separation technique. Recent advances in liquid chromatography separation science, involving the use of multiple HPLC pumps connected through detailed valve-connection systems, allow for the comprehensive separation of complex biological samples to be achieved at a high speed. In the online coupled 2DLC arrangement, the high separation speed of the second chromatography step enables full, and thus comprehensive, separation of all the effluent materials from the first step (22). Reversed-phase liquid chromatography (RPLC) is often employed as the second separation for its high speed and desalting capability. After this step has removed the salts, detergents, and other impurities, effluent peptides can be analyzed directly by a conventional UV detector, which provides a UV trace map of eluted peptides or by electrospray ionization mass spectrometry (ESIMS), which measures the $\mathrm{m} / \mathrm{z}$ ratios of the eluted peptides. In the following sections, we will describe several comprehensive 2DLC systems that have been developed in the past few years.

\section{COUPLED SIZE-EXCLUSION AND RPLC}

Coupled size-exclusion chromatography (SEC) and RPLC have been used in a heart-cutting configuration (1). The SEC phase selects molecules within the desired molecular weight range, and the reversed phase fractionates molecules by hydrophobicity. In 1997, Opiteck et al. reported a comprehensive 2-D SECRPLC technique for peptide mapping (27). Their basic configuration includes six serially connected SEC columns and two parallel RP columns (Figure 1). In this configuration, the sample is injected into the first of the six standard SEC columns (i.d., $7.8 \times 300 \mathrm{~mm}$ ). The effluent materials from the SEC columns are injected into either of one of two parallel RPLC columns (nonporous $\mathrm{C}_{18^{-}}$ modified silica; i.d., $4.6 \times 33 \mathrm{~mm}$ ) by a SEC-RP interface port. Two RPLC columns alternate between loading and analyzing modes in 4-min intervals, al- lowing all the effluent materials from the SEC step to be fractionated by RPLC. At any given time, one of the two parallel RPLC columns is loading and desalting samples from the SEC column, while the other is fractioning samples and eluting directly into a UV detector and a mass spectrometer. The UV detector provides a 2-D PAGE-like chromatogram by plotting UV signals against SEC and RP retention times, and the ESI-MS provides mass information for the eluted peptides.

This system was tested using a tryptic peptide mixture of BSA $(66.4 \mathrm{kDa})$. Using only $5 \mu \mathrm{L}$ tryptic peptide mixture, they observed 48 of the 49 tryptic fragments on the LC-LC 2-D chromatogram in a 3 -h run (27). The mass spectrometer detected and identified 58 peptides, including all 49 tryptic peptides in addition to nine non-tryptic peptides, which resulted in $90 \%$ coverage of the BSA sequence (27).

A modified SEC-RP system was reported from the same laboratory for high-resolution separation of proteins (29). The modifications, aimed at improving separation capacity while staying within the operating pressure of the
HPLC pump, included ( $i$ ) doubling the column length and adding two more columns for the first SEC dimension, (ii) tripling the length of the second dimension RPLC column, and (iii) reducing the column flow rate by $40 \%$. These changes resulted in an $80 \%$ increase in the number of proteins from E. coli cell lysate that were detected on the UV trace map (29). Fourteen proteins separated by this system were recovered and sequenced to validate this method. By combining matrix-assisted laser desorption ionization time-offlight mass spectrometry (MALDITOF-MS) and Edman sequencing, all of the 14 proteins were identified (29).

The SEC-RPLC technique has good reproducibility and protein/peptide recovery. Because RPLC is used as the second separation dimension, the eluted materials are compatible with mass spectrometry, which provides far more information than UV detection. However, SEC causes peak broadening, which invariably leads to sample dilution and overlap, significantly reducing the separation potential of RPLC. It is not surprising that the 2-D chromatogram generated by this method has

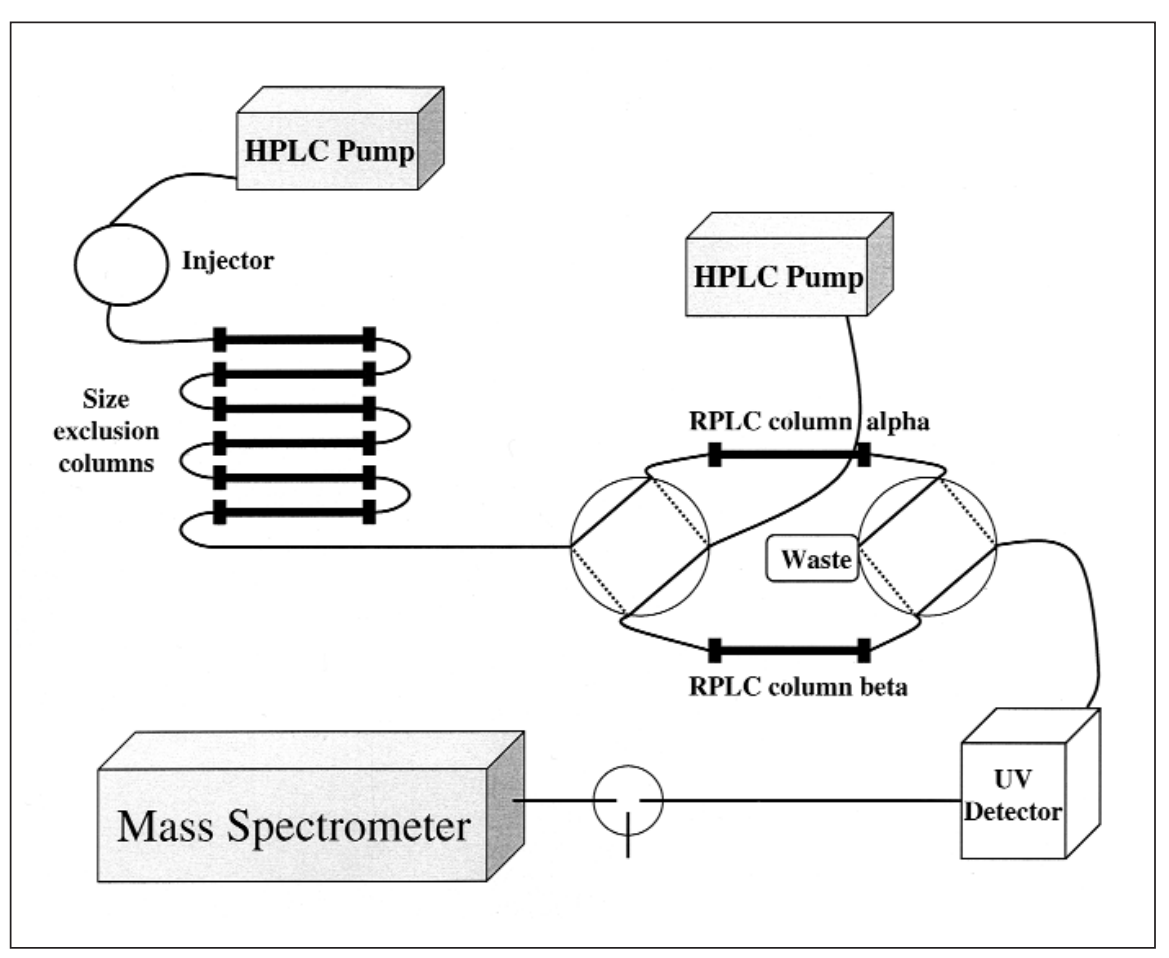

Figure 1. A schematic drawing of the 2DLC-LC-MS system using six SEC columns and two parallel RPLC columns with a dual-detection method in UV and mass spectrometry. This figure was modified based on Reference 27 . 
much lower resolution than that generated by the 2-D PAGE approach. Nonetheless, SEC-RPLC 2DLC produces an orthogonal separation and represents considerable improvements over the 1DLC approaches.

\section{COUPLED ION-EXCHANGE AND RPLC}

In 1997, Opiteck et al. (28) reported a comprehensive coupled ion-exchange chromatography (IEC) and RP chromatography system for the separation of protein mixtures. The configuration of this IEC-RPLC was similar to the 2-D SEC-RPLC system but was simpler. A single strong cation exchange column replaced the serial SEC columns, and, only one RPLC column was necessary (28). In a typical chromatography experiment, a 120-min cation exchange separation was coupled online with 48 individual 150-s RPLC runs, with a total combined run time of $2 \mathrm{~h}$ (28). This was the first report of a LC-LC system coupled online with ESI-MS, allowing the accurate mass determination for eluted proteins. The online linking of liquid chromatography separations with ESIMS allowed extensive automation.

A protein standard containing 10 known components was separated by this system in $2 \mathrm{~h}$ (28). When used to profile the E. coli proteome, a more complete 2-D UV trace map resulted from this method than with the previously described SEC-RPLC system (29). The molecular weight information provided by ESI-MS was used to search the Swiss-Prot database, which yielded lists of possible proteins for each mass (28).

In 2001, Davis et al. (9) reported a simple 2DLC system for comprehensive proteomic analysis of tryptic peptide mixtures. The first separation was strong cation exchange (SCX) chromatography, a variant of IEC. As the unbound and salt-eluted peptides eluted from the IEC column, they were separated by RPLC and analyzed by data-dependent LC-MS-MS (9). In their setup (Figure 2), the RPLC captured materials that did not bind to the IEC during sample loading. While the IEC column was washed, a RPLC run was performed on the IEC-unbound peptides to further separate them by hydrophobicity before mass spectroscopy analysis. The IECbound peptides were then eluted with a single high-salt buffer and separated in the RPLC as before (Figure 2). This method took advantage of the high separation power of RPLC and its compatibility with ion-trap tandem mass spectrometry for peptide sequencing but, unfortunately, did not fully utilize the separation power of IEC. As suggested by the authors, this system was not optimal for highly complex peptide mixtures but was nevertheless a robust method for less complex samples (9).

This method was tested using a complex peptide mixture derived from human tissue samples (9). Proteins were digested with trypsin, and the peptide mixture was then subjected to 2-D IECRPLC separation. Single-dimensional RPLC was performed for comparison. In both cases, the peptides eluted from RPLC were directly injected into LCQITMS for sequence analysis. The 2-D IEC-RPLC method identified 869 peptides, corresponding to 213 proteins, while 609 peptides from 148 proteins appeared in the 1DLC method (9).

Since Opiteck et al. (28) first reported an online coupled LC-LC-MS system, protein identification has shifted to the peptide level. Accurate masses of intact tryptic peptides can be used to make protein identifications through the process of mass mapping. Alternatively, peptides can be matched to database sequences via tandem mass spectrometry (37). There are several reasons why identification at the peptide level is preferable. Posttranslational modifications, often observed in eukaryotic proteins, may increase the observed mass of a protein above its calculated mass, while cellular protease activities may shorten the length of a protein. As result, molecular weight information of proteins obtained from mass spectroscopy may not correspond well with that predicted from gene sequence. Furthermore, unrecognized introns or splice variants for a protein's corresponding gene sequence may complicate the calculation of the accurate mass of the full protein. The recent explosive growth of genomic data has aided protein identification significantly, but that success has been achieved at the level of peptides not intact proteins $(30,38)$.

\section{COUPLED RP AND RPLC}

Most multidimensional separation methods take advantage of the different retention modes of different separation media. In a unique approach, Chen et al. reported a protein separation technique using the different retention properties of RPLC at different temperatures $(7,34)$. In their configuration, the two nonporous RPLC columns were connected in a series. One RPLC column was run at room temperature, while the other was run at $60^{\circ} \mathrm{C}$. The use of the two columns increased the loading capacity by approximately $25 \%$ (from 290 to $360 \mu \mathrm{g}$ ). Moreover, only a small fraction of the total loaded proteins were in the second column at any given time, which in effect increased the separation efficiency of the whole system.

A cell lysate of E. coli was separated by this system in $15 \mathrm{~min}$, and more than 60 peaks were observed (34). There were on average two proteins in each peak, and a total of 120 proteins were recovered. This method was also used to determine the difference in protein expression profiles of $E$. coli cultures grown in a glucose-based and a L-arabinose-based media. It successfully showed that proteins involved in L-arabinose metabolism were only detected in E. coli culture grown in L-arabinose and not in cultures grown in glucose (34).

A limitation of this method was that low-abundance proteins were rarely observed. Theoretically, the coupling of two liquid chromatography separations with the same retention mode cannot increase peak capacity to the same extent as two orthogonal separations. Although more proteins could be loaded onto the pair of columns, an increase in the peak capacity of the separation system was needed to improve the dynamic range of detection.

\section{COUPLED RPLC AND CAPIL- LARY ELECTROPHORESIS}

Capillary electrophoresis (CE) can be paired with tandem mass spectrometry under automated control to separate and to identify peptide mixtures. CE separations feature improved theoretical plates over liquid chromatography, shorter separation times, and lower sam- 
ple consumption. However, the low volume of CE requires that the samples be highly concentrated, which complicates biological analysis. This problem can be circumvented with sample pre-concentration on hydrophobic media, such as solid-phase microextraction (SPE) before $\mathrm{CE}$ fractionation. SPE not only concentrates the samples but also eliminates salts, detergents, buffers, and other small-molecule impurities $(15,23)$.

One example of LC-CE reported by Tong et al. used this method to analyze the protein components of the yeast ribosome (33). The experiment used a complex procedure for LC-CE-MS (Figure 3). First, the sample was digested and acidified. The resulting peptides were pre-concentrated in an SPE cartridge containing $\mathrm{RP} \mathrm{C}_{18}$ packing material. Eleven increasing concentrations of hydrophobic solvent were used to "step" the peptides off of the cartridge and into the capillary. The capillary, which had been coated to reduce peak broadening and tailing, conducted a 30 min, reduced-voltage CE separation. As peptides emerged from the capillary, they were directly electrosprayed into a mass spectrometer.

This technique was also used in analyzing the ribosome complex of Saccharomyces cerevisiae, which contained approximately 75 major proteins (33). A search of collected MS-MS data against the yeast open reading frame database by SEQUEST (10) identified a total of 66 proteins through 136 unique peptides. A control experiment without the multi-step elution yielded only 13 proteins. The step elution method made this 2-D separation method more effective for analyzing complex samples. While this technique was not completely automated, the authors indicated that commercial $\mathrm{CE}$ instruments might make this possible (33).

In contrast to the LC-CE method described earlier, a "traditional" slowfirst-phase, fast-second-phase multidimensional LC-CE method was developed in Jorgenson's laboratory $(4,17,20,21)$. As the first phase in their RPLC-CE configuration (17), a Zor$\mathrm{bax}^{\circledR}{ }^{\circledR} \mathrm{SB}-\mathrm{C}_{8}$ analytical column (Agilent Technologies, Palo Alto, CA, USA) that is connected with the capillary through an eight-port electrically actuated valve and a stainless tee is used. Horse heart cytochrome $c$ was digested with trypsin and derivatized with a fluorescent reagent for a test of this system. The result showed a 2-D-PAGElike image by recording the fluorescence of peptides eluting at both RPLC and CE retention times. Al-

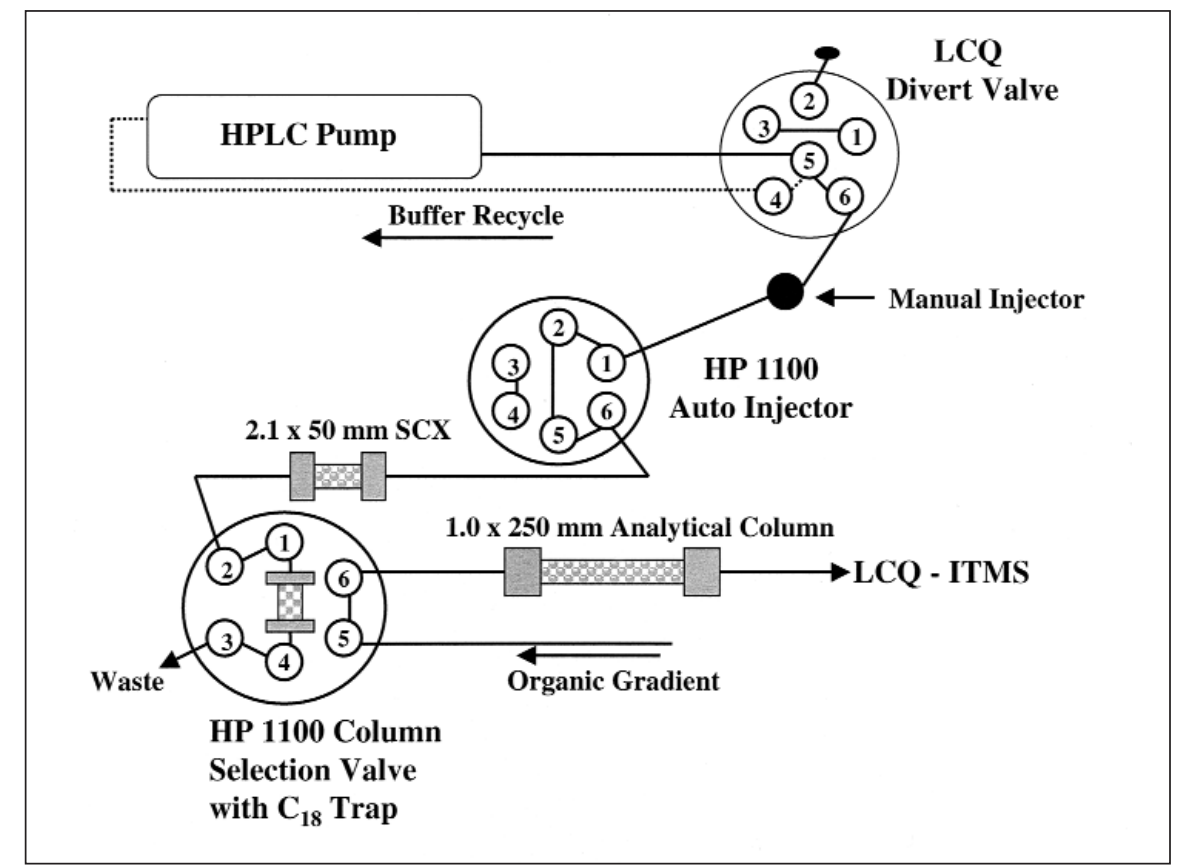

Figure 2. A flow scheme in a multidimensional LC-MS platform. The dual-pump configuration includes a SCX column and an RP analytical column. This figure was modified based on Reference 9. 


\section{Proteomic Technologies}

though the RPLC retention time shifted from run to run, the peak pattern was reproducible. Similar designs were also applied to 2-D SEC-CE (17), 2-D RPLC-fast CE (21), and 3-D SECRPLC-CE systems (20). Jorgenson's laboratory was the first to use comprehensive online coupled LC-CE to analyze complex peptide mixtures. However, the detection method could not provide sufficient information for unknown protein identification.

\section{IMMOBILIZED METAL AFFINITY CHROMATOGRAPHY COUPLED WITH CE}

Protein phosphorylation and dephosphorylation events are significant in many cellular processes, such as enzyme regulation and signal transduction pathways $(8,16)$. Studying these modifications is a difficult task since phosphorylated proteins in cells are less concentrated than nonphosphorylated ones (5). Most research into these proteins focuses on determining the precise location(s) of phosphorylation and on quantifying the relative amounts of modified and unmodified forms.

The concentration of phosphorylated peptides can be enhanced by pre-enrichment before analysis by tandem mass spectrometry. Immobilized metal affinity chromatography (IMAC) when used with $\mathrm{Fe}^{3+}$ or $\mathrm{Ga}^{3+}$ has the ability to concentrate and to enrich phosphorylated proteins and peptides $(2,24,25)$. Since these peptides are often isolated in small quantities, a sensitive detection method is required for subsequent analysis (5). $\mathrm{CE}$, because of its potential for high sensitivity and resolution, is often used as the separation following IMAC.

An online IMAC-CE-ESI-MS separation technique to analyze phosphorylated peptides was first reported by Cao and Stults in 1999 (5), and a follow-up paper was published in 2000 (6). In their configuration (Figure 4), phosphopeptides were first concentrated by the IMAC and then by an immediate CE phase in the same capillary for rapid separation by charge (5). CEfractionated peptides were then injected into ESI-MS, and tandem mass spectrometry revealed the phosphorylation sites and peptide sequences.
To test this method, they used synthetic peptides containing phosphorylated tyrosine residues and tryptic peptides from human angiotensin II. Results showed that $\mathrm{Fe}^{3+}$-IMAC affinity columns retain and concentrate phosphopeptides at amounts as low as $28 \mathrm{fmol}$ (5). They used SDS-PAGE for the isolation of phosphorylated proteins of interest from a protein mixture before in-gel

trypsin digestion and IMAC-CE-ESIMS analysis for phosphopeptide mapping. Using $\beta$-casein, they determined the minimum amount of starting protein needed to get acceptable mass spectroscopy signals for MS-MS sequencing (6). The lower limit for protein gel loading in this case was $500 \mathrm{fmol}(6)$. This finding demonstrated that approximately 20 -fold more protein is needed

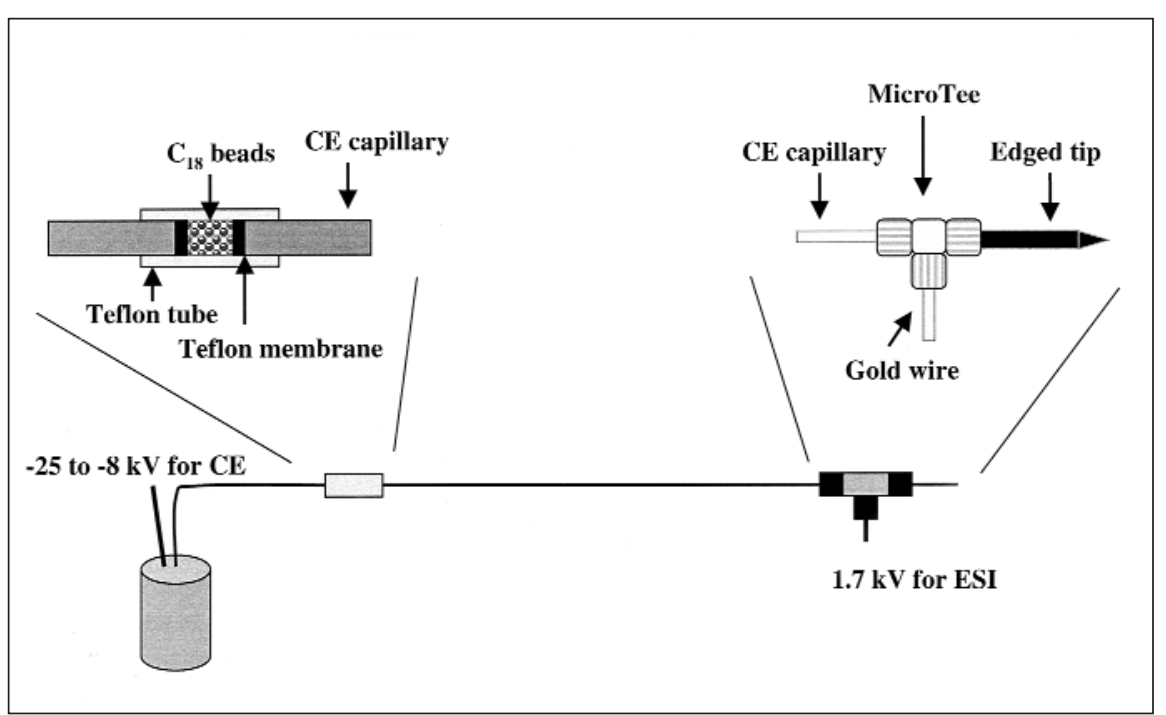

Figure 3. A design of SPE coupled with CE-MS for peptide analysis. The SPE is used for rapid RP elution and CE for the separation of peptides. A custom-pulled capillary tip is used for electrospray ionization for mass spectroscopy analysis. This figure was modified based on Reference 33 .

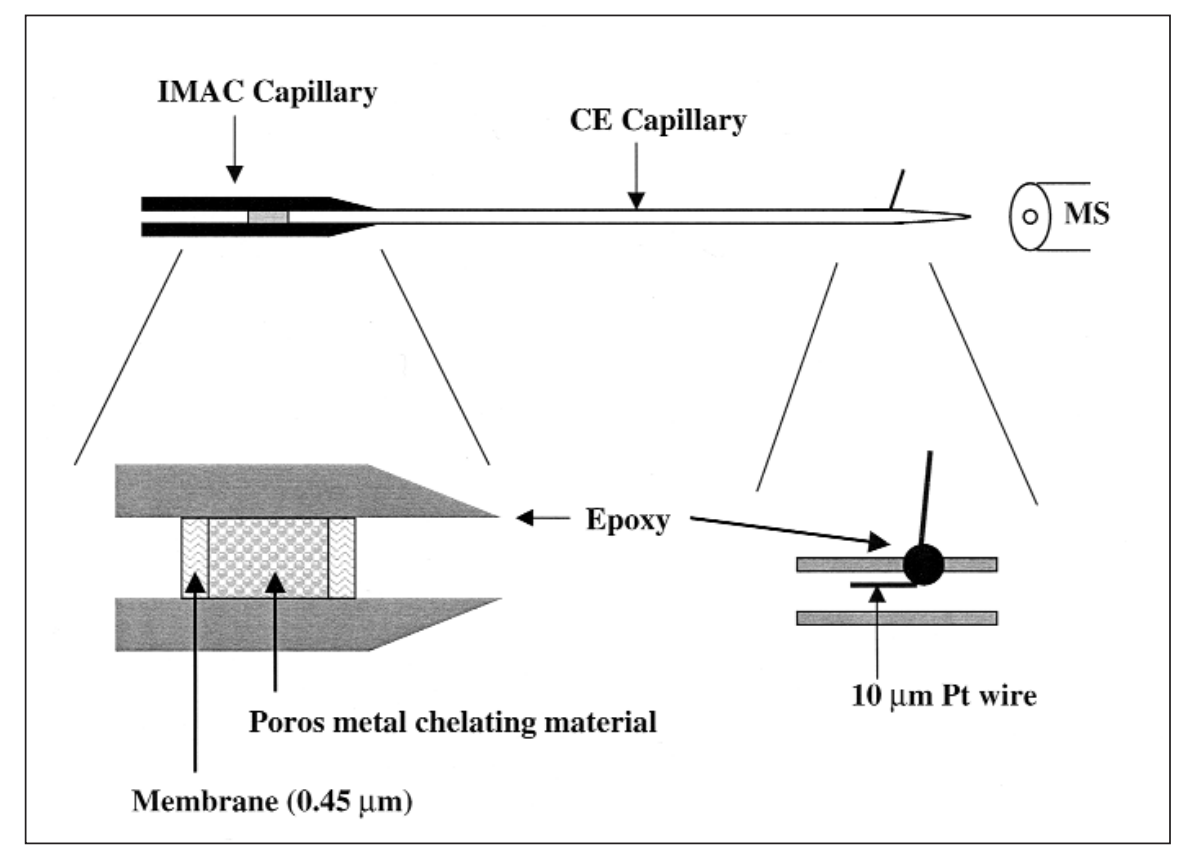

Figure 4. A schematic of the online IMAC-CE-ESI-MS apparatus. IMAC phase traps the phosphopeptides, and CE is used for rapid separation. This figure was modified based on Reference 5. 
when IMAC-CE-ESI-MS is preceded by SDS-PAGE. As we have indicated, this could be due to poor peptide recovery from polyacrylamide gels (6).

Although several phosphorylated peptides were identified $(5,6)$, separation by $\mathrm{CE}$ was not ideal. IMAC as the first phase enhanced the concentration limits of detection but decreased CE resolution, yielding to broader peaks. For less complex samples, the decrease in CE resolution was compensated for by tandem mass spectrometry. This approach may be best suited for the analysis of relatively pure phosphorylated proteins isolated from gel electrophoresis.

In addition to the described online IMAC-CE method, Figeys et al. (13) reported a method for mapping phosphoproteins using offline IMAC purification and enrichment, followed by solid-phase extraction, capillary zone electrophoresis, and tandem mass spectrometry analysis.

\section{MULTIDIMENSIONAL PROTEIN IDENTIFICATION TECHNOLOGY}

A high-throughput method for protein identification from very complex mixtures has been demonstrated in a process called MudPIT $(19,35)$. In 1999, Link et al. (19) reported an improved technique using a biphasic column (a column containing two different packing materials) and multiple steps of chromatography for improved detection sensitivity and nearly complete automation. The technique was demonstrated by analyzing peptides derived from the digestion of the ribosomal protein complex and total yeast cell lysate.

The biphasic MudPIT column achieved three major advantages. The column used SCX resin and $\mathrm{RP} \mathrm{C}_{18}$ in a fused silica capillary column (Figure 5A). By using a column with an i.d. of $100 \mu \mathrm{m}$, the flow rate was decreased; thus, the sensitivity of MudPIT was increased. In addition, because SCX separated by charge and RP separated by hydrophobicity, the two dimensions of chromatography were orthogonal. The unique biphasic column effectively eliminated the valves and dead volume commonly associated with complex chromatography. These advantages allowed MudPIT to be capable of separating samples of greater complexity than otherwise possible.

The chromatography process for MudPIT involved a series of increasing salt steps, with a ramp of organic solvent between each step to elude the peptides that moved to the RP phase. Approximately 2 min were required for each salt step, and each RP gradient took approximately $80 \mathrm{~min}$. As peptides eluted from the RPLC, they flowed directly into the electrospray interface of a tandem mass spectrometer (Figure 5B). Data collected during the experiment were submit- 
ted to the SEQUEST algorithm for peptide identification and to summarizing software for assembling protein identifications from SEQUEST results.

Using a modified MudPIT approach, Washburn and co-workers (35) identified a total of 1484 proteins from a cell lysate sample of $S$. cerevisiae, including low-abundance proteins, proteins with extreme $\mathrm{pI}$ values, and even many proteins containing several putative transmembrane domains. The total number of proteins identified represented a dynamic range of at least 10000:1 (36).

MudPIT is a highly developed experimental methodology, but there are limitations to the technique. The column packing and sample loading procedures are entirely manual, which limits its sample throughput. Each experiment may take a substantial amount of instrument time, depending on the number of salt steps used. The number of spectra produced by the experiment is in the tens of thousands, placing heavy demands on data storage and processing capability. Improved tools for summarizing SEQUEST results will become necessary as these experiments increase in scale (32).

\section{CONCLUSION}

All the multidimensional separation techniques reviewed here provided better peak capacity and higher capability of analyzing complex protein mixtures than the 1-D approach. Many of these techniques, except for the MudPIT and CE methods, use two columns and couple them via a switch valve. The common limitation of all of these methods is largely sample detection (mostly mass spectrometry) rather than chromatographic separations. However, today's mass spectrometers have characteristics of high throughput, broad dynamic range, and great sensitivity. Because a mass spectrometer is both a separation and detection tool, when it is directly connected to 2-D separation apparatus, it serves as a third separation dimension and a detector, as shown in

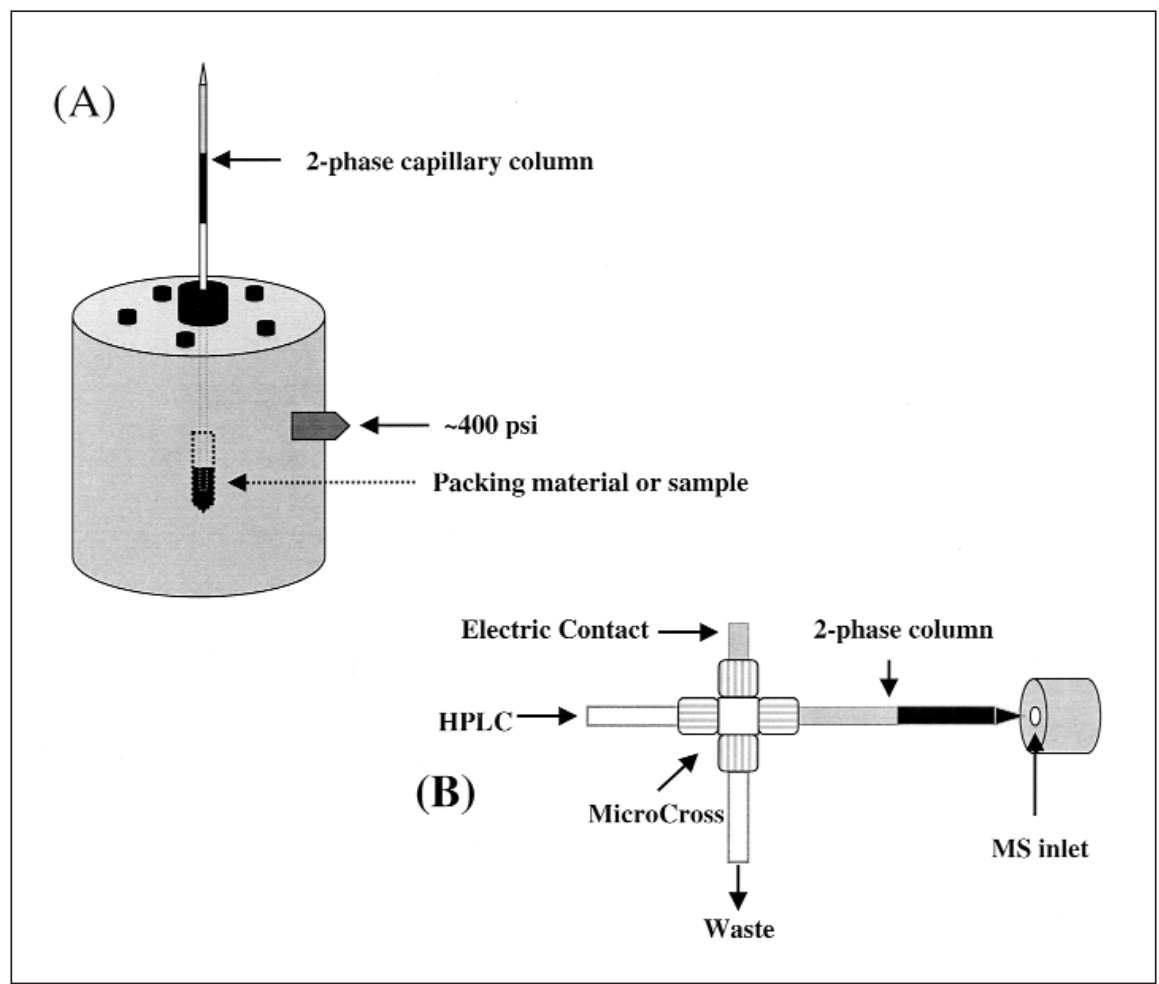

Figure 5. The construction of a 2DLC column and its interface with mass spectrometry. (A) A pressure bomb is used for column packing and sample loading. (B) The flow rate of in the 2-D column is controlled at $100-300 \mathrm{~nL} / \mathrm{min}$, and ESI is achieved by applying $2 \mathrm{kV}$ to the gold wire. This figure was modified based on Reference 35 and Yates, J.R. 1998. Peptide sequencing by tandem mass spectrometry, p. 529-538. In Cell Biology: A Laboratory Handbook. Academic Press, New York.

MudPIT. Because of its high-throughput protein identification potential and automation, MudPIT offers a great alternative to the "traditional" 2-D gel electrophoresis and mass spectrometry approach in proteomic research (18).

Through the current examples of multidimensional liquid chromatography in combination with mass spectrometry, it is clear that there are tremendous potentials to create highly sensitive, automated systems. Multidimensional liquid separations offer the high capacity for protein separations in proteomic applications. One may expect to see more such applications and developments in the future.

\section{ACKNOWLEDGMENTS}

The authors thank Dr. Claire Delahunty, Meiling Shang, and David Tabb for providing valuable editorial comments on the manuscript. The authors acknowledge support from the Office of Naval Research grant no. N00014-00-10421, National Institutes of Health grant no. R33CA81665-01, and University of Washington grant no. RR11823-05.

\section{REFERENCES}

1.Amari, J.V. and I. Mazsaroff. 1996. Analysis of recombinant human interleukin-11 fusion protein derived from E. coli lysate by combined size-exclusion and reversed-phase liquid chromatography. J. Chromatogr. A 729:113-124.

2.Andersson, L. and J. Porath. 1986. Isolation of phosphoproteins by immobilized metal $\left(\mathrm{Fe}^{3+}\right)$ affinity chromatography. Anal. Biochem. 154:250-254.

3.Berkowitz, S.A. 1987. Linear multidimensional liquid chromatography in the preparative scale purification of calmodulin from brain extract. Anal. Biochem. 164:254-260.

4.Bushey, M.M. and J.W. Jorgenson. 1990. Automated instrumentation for comprehensive 2-dimensional high-performance liquidchromatography capillary zone electrophoresis. Anal. Chem. 62:978-984.

5.Cao, P. and J.T. Stults. 1999. Phosphopeptide analysis by on-line immobilized metal-ion affinity chromatography-capillary electrophoresis-electrospray ionization mass spectrometry. J. Chromatogr. A 853:225-235.

6.Cao, P. and J.T. Stults. 2000. Mapping the phosphorylation sites of proteins using on-line immobilized metal affinity chromatography/ capillary electrophoresis/electrospray ionization multiple stage tandem mass spectrometry. Rapid Commun. Mass Spectrom. 14:16001606. 
7.Chen, Y., D. Wall, and D.M. Lubman. 1998. Rapid identification and screening of proteins from whole cell lysates of human erythroleukemia cells in the liquid phase, using non-porous reversed phase high-performance liquid chromatography separations of proteins followed by matrix-assisted [correction of multi-assisted] laser desorption/ionization mass spectrometry analysis and sequence database searching. Rapid Commun. Mass Spectrom. 12:1994-2003.

8.Cohen, P. 1992. Signal integration at the level of protein kinases, protein phosphatases and their substrates. Trends Biochem. Sci. 17:408413.

9.Davis, M.T., J. Beierle, E.T. Bures, M.D. McGinley, J. Mort, J.H. Robinson, C.S. Spahr, W. Yu et al. 2001. Automated LC-LCMS-MS platform using binary ion-exchange and gradient reversed-phase chromatography for improved proteomic analyses. J. Chromatogr. B 752:281-291.

10.Eng, J.K., A.L. McCormack, and J.R. Yates. 1994. An approach to correlate tandem mass spectral data of peptides with amino acid sequences in a protein database. J. Am. Soc. Mass Spectrom. 5:976-989.

11.Fichmann, J. and R. Westermeier. 1999. 2D protein gel electrophoresis. An overview, p. 1-7. In A.J. Link (Ed.), 2-D Proteome Analysis Protocol. Humana Press, Totowa, NJ.

12.Fields, S. 2001. Proteomics. Proteomics in genomeland. Science 291:1221-1224.

13.Figeys, D., S.P. Gygi, Y. Zhang, J. Watts, M. Gu, and R. Aebersold. 1998. Electrophoresis combined with novel mass spectrometry techniques: powerful tools for the analysis of proteins and proteomes. Electrophoresis 19:18111818.

14.Giddings, J.C. 1987. Concepts and comparisons in multidimensional separation. J. High Resolut. Chromatogr. Commun. 10:319-323.

15.Hunt, D.F., R.A. Henderson, J. Shabanowitz, K. Sakaguchi, H. Michel, N. Sevilir, A.L. Cox, E. Appella et al. 1992. Characterization of peptides bound to the class I MHC molecule HLA-A2.1 by mass spectrometry. Science 255:1261-1263.

16.Hunter, T. 2000. Signaling-2000 and beyond. Cell 100:113-127.

17.Larmann, J.P., A.V. Lemmo, A.W. Moore, and J.W. Jorgenson. 1993. Two-dimensional separations of peptides and proteins by comprehensive liquid chromatography-capillary electrophoresis. Electrophoresis 14:439-447.

18.Lin, D., A.J. Alpert, and J.R. Yates, 3rd. 2001. Multidimensional protein identification technology as an effective tool for proteomics. Am. Genomic/Proteomic Technol. 1:38-46.

19.Link, A.J., J. Eng, D.M. Schieltz, E. Carmack, G.J. Mize, D.R. Morris, B.M. Garvik, and J.R. Yates, 3rd. 1999. Direct analysis of protein complexes using mass spectrometry. Nat. Biotechnol. 17:676-682

20.Moore, A.W. and J.W. Jorgenson. 1995. Comprehensive three-dimensional separation of peptides using size exclusion chromatography/reversed phase liquid chromatography/ optically gated capillary zone electrophoresis. Anal. Chem. 67:3456-3463.

21.Moore, A.W. and J.W. Jorgenson. 1995. Rapid comprehensive two-dimensional sepa- rations of peptides via RPLC-optically gated capillary zone electrophoresis. Anal. Chem. 67:3448-3455.

22.Moore, A.W., J.P. Larmann, A.V. Lemmo, and J.W. Jorgenson. 1996. Two-dimensional liquid chromatography-capillary electrophoresis techniques for analysis of proteins and peptides. Methods Enzymol. 270:401419.

23.Moseley, M.A., L.J. Deterding, K.B. Tomer, and J.W. Jorgenson. 1991. Determination of bioactive peptides using capillary zone electrophoresis/mass spectrometry. Anal. Chem. 63:109-114.

24.Muszynska, G., L. Andersson, and J. Porath. 1986. Selective adsorption of phosphoproteins on gel-immobilized ferric chelate. Biochemistry 25:6850-6853.

25.Neville, D.C., C.R. Rozanas, E.M. Price, D.B. Gruis, A.S. Verkman, and R.R. Townsend. 1997. Evidence for phosphorylation of serine 753 in CFTR using a novel metal-ion affinity resin and matrix-assisted laser desorption mass spectrometry. Protein Sci. 6:2436-2445.

26.Nilsson, C.L. and P. Davidsson. 2000. New separation tools for comprehensive studies of protein expression by mass spectrometry. Mass Spectrom. Rev. 19:390-397.

27.Opiteck, G.J., J.W. Jorgenson, and R.J. Anderegg. 1997. Two-dimensional SEC/RPLC coupled to mass spectrometry for the analysis of peptides. Anal. Chem. 69:2283-2291.

28.Opiteck, G.J., K.C. Lewis, J.W. Jorgenson, and R.J. Anderegg. 1997. Comprehensive on-line LC/LC/MS of proteins. Anal. Chem. 69:1518-1524.

29.Opiteck, G.J., S.M. Ramirez, J.W. Jorgenson, and M.A. Moseley. 1998. Comprehensive two-dimensional high-performance liquid chromatography for the isolation of overexpressed proteins and proteome mapping. Anal. Biochem. 258:349-361.

30.Pandey, A. and M. Mann. 2000. Proteomics to study genes and genomes. Nature 405:837846.

31.Regnier, F. and G. Huang. 1996. Future potential of targeted component analusis by multidimensional liquid chromatography-mass scpectrometry. J. Chromatogr. A 750:3-10.

32.Tabb, D.L., W.H. McDonald, and J.R. Yates, 3rd. 2002. DTASelect and contrast: tool for assembling and comparing protein identification from shotgun proteomics. J. Proteome Res. 1:21-26.

33.Tong, W., A. Link, J.K. Eng, and J.R. Yates, 3rd. 1999. Identification of proteins in complexes by solid-phase microextraction/multistep elution/capillary electrophoresis/tandem mass spectrometry. Anal. Chem. 71:22702278.

34.Wall, D.B., D.M. Lubman, and S.J. Flynn. 1999. Rapid profiling of induced proteins in bacteria using MALDI-TOF mass spectrometric detection of nonporous RP HPLC-separated whole cell lysates. Anal. Chem. 71:38943900.

35.Washburn, M.P., D. Wolters, and J.R. Yates, 3rd. 2001. Large-scale analysis of the yeast proteome by multidimensional protein identification technology. Nat. Biotechnol. 19:242-247.
36.Wolters, D.A., M.P. Washburn, and J.R. Yates, 3rd. 2001. An automated multidimensional protein identification technology for shotgun proteomics. Anal. Chem. 73:56835690 .

37.Yates, J.R., 3rd. 1998. Database searching using mass spectrometry data. Electrophoresis 19:893-900.

38.Yates, J.R., 3rd. 1998. Mass spectrometry and the age of the proteome. J. Mass Spectrom. 33:1-19.

39.Yates, J.R., 3rd. 2000. Mass spectrometry. From genomics to proteomics. Trends Genet. 16:5-8.

Address correspondence to:

Dr. John R. Yates III

Department of Cell Biology

The Scripps Research Institute

La Jolla, CA 92037, USA

e-mail: jyates@scripps.edu

For reprints of this or any other article, contact Reprints@BioTechniques.com 